\title{
Spontaneous Growth of Gallium-Filled Microcapillaries on Ion-Bombarded GaN
}

\author{
Aurelien Botman \\ FEI Company, 5350 Northeast Dawson Creek Drive, Hillsboro, Oregon 97214-5793, USA \\ Alan Bahm \\ FEI Company, 5350 Northeast Dawson Creek Drive, Hillsboro, Oregon 97214-5793, USA \\ School of Physics and Advanced Materials, University of Technology, \\ Sydney, Post Office Box 123, Broadway, New South Wales 2007, Australia \\ Steven Randolph and Marcus Straw \\ FEI Company, 5350 Northeast Dawson Creek Drive, Hillsboro, Oregon 97214-5793, USA
}

Milos Toth*

School of Physics and Advanced Materials, University of Technology, Sydney, Post Office Box 123, Broadway, New South Wales 2007, Australia

(Received 2 May 2013; revised manuscript received 4 July 2013; published 25 September 2013)

\begin{abstract}
Bottom-up growth of microscopic pillars is observed at room temperature on GaN irradiated with a $\mathrm{Ga}^{+}$beam in a gaseous $\mathrm{XeF}_{2}$ environment. Ion bombardment produces Ga droplets which evolve into pillars, each comprised of a spherical Ga cap atop a Ga-filled, gallium fluoride tapered tube (sheath). The structures form through an interdependent, self-ordering cycle of liquid cap growth and solid sheath formation. The sheath and core growth mechanisms are not catalytic, but instead consistent with a model of ion-induced $\mathrm{Ga}$ and $\mathrm{F}$ generation, Ga transport through surface diffusion, and heterogeneous sputtering caused by self-masking of the tapered pillars.
\end{abstract}

Emergent phenomena such as spontaneous pattern formation, self-assembly, and self-organization [1-3] have stimulated much research into the underlying mechanisms, and applications in bottom-up growth [3] at length scales ranging from the atomic to macroscopic. Here, we report a spontaneous, room temperature growth mechanism that yields microscopic pillars each comprised of a solid, tapered, gallium fluoride sheath and a Ga core that protrudes from the sheath and forms a liquid spherical cap at the pillar tip. The growth process was observed on $\mathrm{GaN}$ irradiated by $\mathrm{a} \mathrm{Ga}^{+}$beam in a gaseous $\mathrm{XeF}_{2}$ environment. Pillar growth is initiated by the formation of a spherical liquid Ga droplet and concurrent growth of a solid sheath, caused by chemical conversion of liquid Ga to gallium fluoride. Tapered pillars emerge from an interdependent, self-ordering cycle of Ga droplet (i.e., pillar cap) growth and sheath formation. The underlying mechanisms are noncatalytic and physically distinct from others reported in the literature, such as vaporliquid-solid, solid-liquid-solid, and solution-liquid-solid growth [4].

It is well known that ion bombardment in vacuum of III-V semiconductors such as GaN [5-7], GaAs, InN, InAs, and InP can give rise to the formation of metallic droplets on the substrate surface $[2,8,9]$. Preferential sputtering of the group $\mathrm{V}$ element and ion beam induced decomposition and restructuring of the surface cause the group III species to accumulate $[5,10,11]$, and droplets to form through nucleation, growth and ripening mechanisms $[2,8,9,12]$.
In some instances, the droplets have very narrow size distributions, and can be ordered through spontaneous or directed processes such as off-normal ion irradiation [2] and prepatterning of droplet nucleation sites [8].

Droplets generated by the irradiation of $\mathrm{GaN}$ in vacuum by a normal incidence, focused, rastered, $30 \mathrm{keV} \mathrm{Ga}{ }^{+}$ beam [13] are shown in Figs. 1(a) and 1(b). The droplets nucleate preferentially at surface steps [Fig. 1(b)],
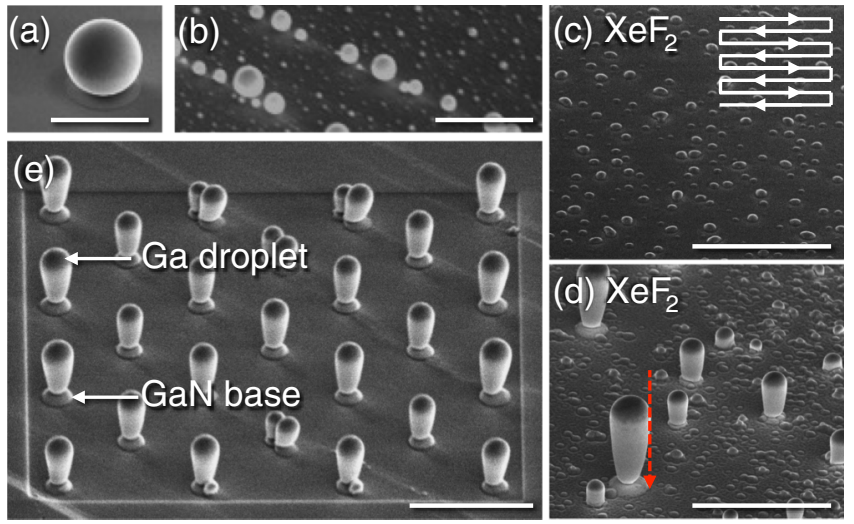

FIG. 1 (color online). (a)-(b) Electron images of Ga droplets on $\mathrm{GaN}$ formed during ion beam irradiation in vacuum, and two frames from a movie of droplets (c) growing to form pillars (d) in $\mathrm{XeF}_{2}$. (e) Pillar array grown on a prepatterned GaN substrate. Each scale bar represents $5 \mu \mathrm{m}$. The ion beam was scanned in the serpentine pattern shown in (c). 
consistent with a mechanism that involves diffusion and pinning at free-energy-minimizing loci. In a gaseous $\mathrm{XeF}_{2}$ environment, ion irradiation also gives rise to the formation of $\mathrm{Ga}$ droplets. However, the droplets act as nucleation sites for the formation of pillars that grow antiparallel to the ion beam. Figs. 1(c) and 1(d) show two frames from Video 1 of the Supplemental Material [13], a movie produced by real time secondary electron imaging of the growth process. The movie reveals that the droplets nucleate in random locations and that the growth of each individual pillar starts, decelerates, and terminates spontaneously as the ion beam is scanned repeatedly over the substrate.

The observed pillar growth is highly atypical in that $\mathrm{XeF}_{2}$ normally acts as a precursor for chemical etching of ion-irradiated substrates [7]. $\mathrm{XeF}_{2}$ is also known to play a role in swelling caused by ion irradiation, and top-down (subtractive) structure formation caused by heterogeneous etching $[6,14]$. Such processes are, however, clearly different from the pillar growth reported here.

The pillars in Fig. 1(d) are aligned vertically, but distributed randomly along the substrate. Ordered arrays [Fig. 1(e)] and individual pillars (Fig. 2) can be fabricated by prepatterning $\mathrm{Ga}$ droplet nucleation sites using the focused $\mathrm{Ga}^{+}$beam. The time evolution of a single pillar is demonstrated in Fig. 2 by 9 frames from Video 2 of the Supplemental Material [13], a movie of the growth process. The images show [6 s]: a hole that had been made by a stationary $\mathrm{Ga}^{+}$beam and acts as a droplet nucleation site; [6-31 s]: droplet nucleation and growth occurring while the ion beam is scanned repeatedly over the rectangle seen in the images; [80-524 s]: pillar formation; [ $400 \mathrm{~s}]$ : droplet nucleation at the edge of the scan rectangle; [400-554 s] pillar growth termination; and [554 s]: droplet nucleation and growth observed to occur at the pillar base
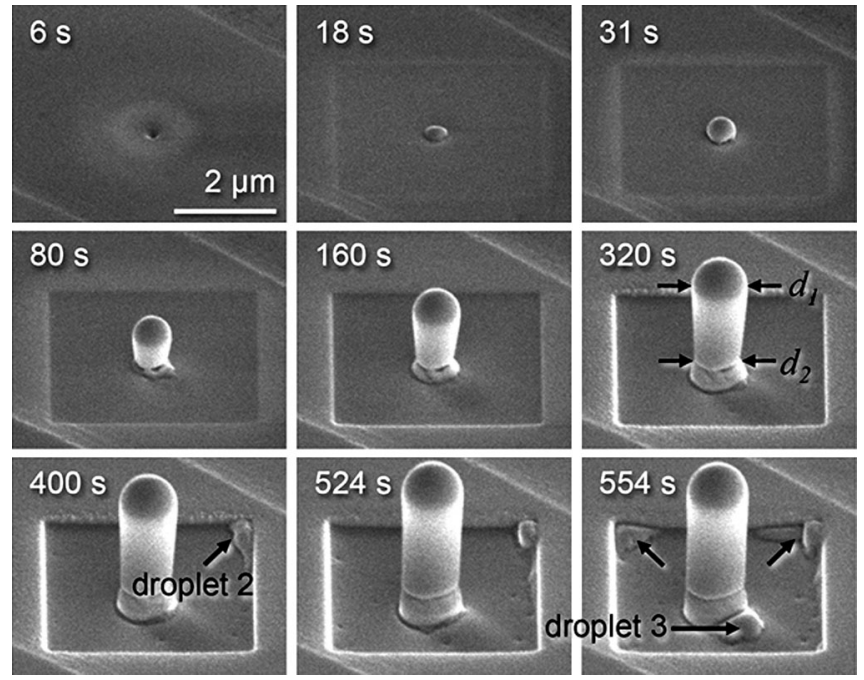

FIG. 2. Electron image sequence showing the growth of a pillar and the formation of secondary Ga droplets. and the edge of the scan rectangle immediately after pillar growth has terminated. Figure 3(a) shows plots of the pillar cap diameter $d_{1}$, the corresponding volume $V$, and the pillar base diameter $d_{2}$ as a function of time $(t)$. Vertical growth terminated at $\sim 530 \mathrm{~s}$, where $\partial V / \partial t=0$.

Pillar composition and internal structure were determined by cross-sectional electron imaging and energy dispersive $x$-ray spectroscopy (EDS). EDS maps of pillars that had been grown for 60,420 , and $1200 \mathrm{~s}$ are shown in Figs. 4(a)-4(c), where each time stamp corresponds to the growth time of a different pillar. Ga and $\mathrm{F}$ (but no Xe, N, or $\mathrm{O})$ were detected in the pillars. The maps show that each pillar is a Ga-filled, gallium fluoride microcapillary (i.e., a tapered fluorinated sheath). The most stable gallium fluoride stoichiometry is $\mathrm{GaF}_{3}$ [15], which has a high melting point of $800^{\circ} \mathrm{C}$, in contrast to that of $\mathrm{Ga}\left(30^{\circ} \mathrm{C}\right)$ which behaves as a supercooled liquid at room temperature. The Ga core extends beyond the sheath and forms a spherical Ga cap [shown in Fig. 4(d)]. The liquid nature of the Ga caps is illustrated by the image sequence in Fig. 5 which shows the coalescence of two adjacent pillar caps into a single, asymmetric Ga droplet. The images also illustrate the high cohesive energy of Ga which is responsible for droplet formation.

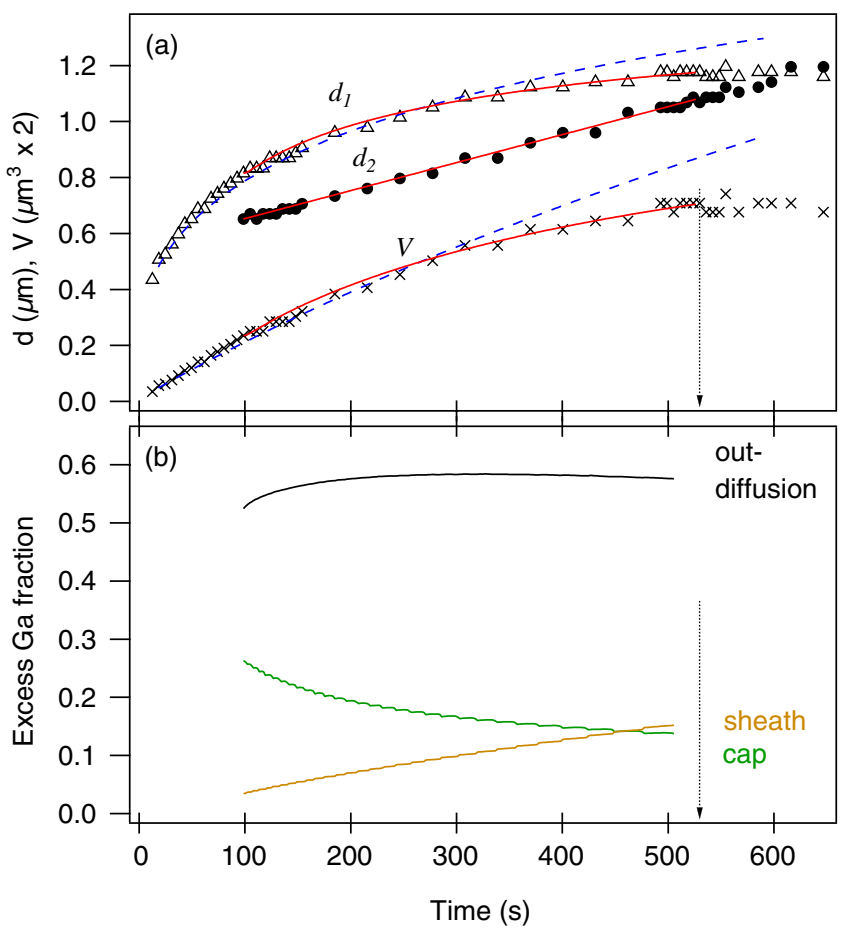

FIG. 3 (color online). (a) Cap volume $V$ (cross), cap diameter $d_{1}$ (triangle), and pillar base diameter $d_{2}$ (filled circle) measured as a function of time, and corresponding curves calculated using Eq. (1) (blue dashed lines) and Eq. (2) (solid lines). (b) Fraction of excess $\mathrm{Ga}$ atoms diffusing into the sheath, the cap, and away from the pillar ("out diffusion") calculated as a function of time. Vertical arrows show the time at which pillar growth terminated. 


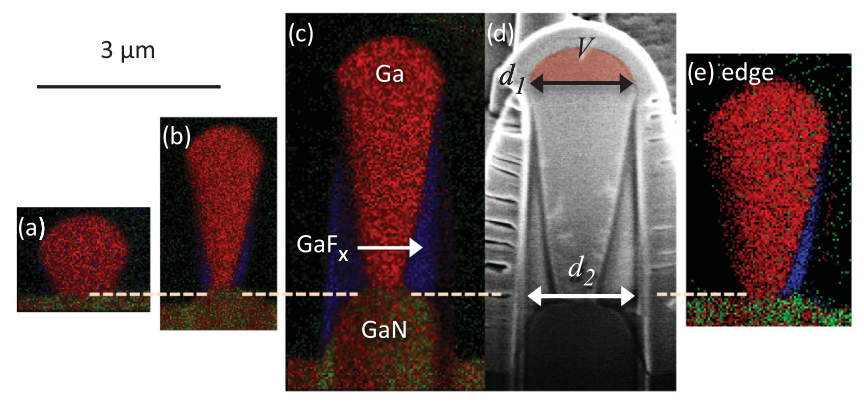

FIG. 4 (color). Cross-sectional compositional maps $(\mathrm{Red}=$ $\mathrm{Ga}$, blue $=\mathrm{F}$, green $=\mathrm{N}$ ) of pillars that had been grown for (a) $60 \mathrm{~s}$, (b) $420 \mathrm{~s}$, and (c) $1200 \mathrm{~s}$, and (d) a secondary electron image of pillar (c). Map (e) shows a pillar grown at the edge of the area bombarded by $\mathrm{Ga}^{+}$ions. The dashed horizontal line shows the position of the GaN surface prior to ion irradiation. Each pillar is coated with a protective Pt film used to minimize cross-sectioning artifacts. The scale bar applies to all images.

Fluorine was detected only in the tapered sheaths [Fig. 4(c)]. The sheaths are present only in regions that were protected from sputtering through masking by the $\mathrm{Ga}$ caps, as indicated by an arrow in Fig. 1(d). Masking also prevents sputtering of the underlying GaN substrate, resulting in the formation of the raised $\mathrm{GaN}$ features seen at the base of each pillar in Figs. 1(e) and 4(c).

Figure 4(e) shows an EDS map of a pillar grown at the periphery of the rectangle scanned by the ion beam. The sheath is present only on the pillar surface that faces the scan rectangle, showing that the ion beam plays a direct role in sheath formation, and that $\mathrm{F}$ is immobile at length scales on the order of the pillar diameter. The map also shows that $\mathrm{F}$ is not entering the pillar from the gas phase (through the cap) and is not causing fluoride growth at the sheath-core interface through a vapor-liquid-solid mechanism.

The compositional maps in Fig. 4 show how Ga droplets evolve into pillar caps and cores, and how sheath formation is enabled by the sidewall taper which prevents sheath removal through sputtering. The maps also indicate how vertical pillars emerge from a cycle of cap growth and sheath formation. However, the maps do not explain the

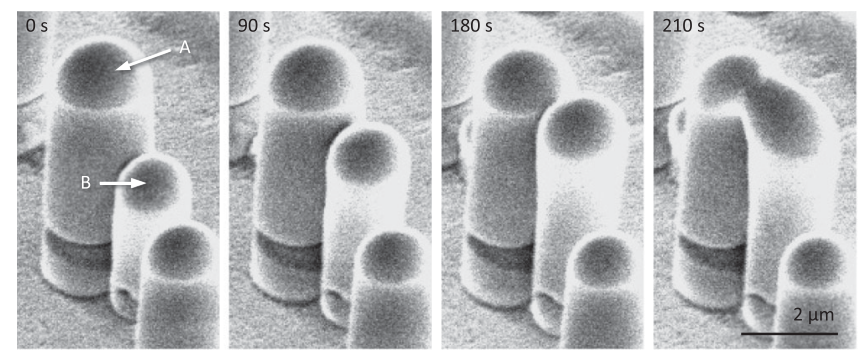

FIG. 5. Image sequence showing a pillar (A) that has reached maximum height and is no longer growing, the growth of an adjacent pillar (B), and coalescence of the pillar caps into a single, asymmetric Ga droplet. pillar growth kinetics. To elucidate the observed growth behavior, we consider the isolated pillar imaged in Fig. 2. The growth rates of the Ga cap volume $(V)$ and diameter $\left(d_{1}\right)$ decrease with time, while the pillar base diameter $\left(d_{2}\right)$ scales linearly with time [see Fig. 3(a)]. This indicates that the growth is rate limited by the supply rate of Ga rather than fluorine. Therefore, we develop a model of growth kinetics based on excess $\mathrm{Ga}$ generation and consumption in the system.

In the limit of short growth times, the sheath volume is negligible, and we assume that (i) the excess Ga generation rate is proportional to the area of $\mathrm{GaN}$ that is bombarded by ions and acts as a source of excess $\mathrm{Ga}$, (ii) a fixed fraction of the excess Ga flows into and is consumed by the pillar, which is located in the center of the Ga source, and (iii) $\mathrm{Ga}$ is removed from the cap through sputtering at a rate that is proportional to the cap surface area, $A_{s}$. We can, therefore, approximate the rate of change of the cap volume, $\partial V / \partial t$, by

$$
\frac{\partial V}{\partial t} \approx \frac{\partial V_{0}}{\partial t}\left(1-\frac{A_{d 1}(t)}{A_{I}}\right)-\frac{\partial \gamma}{\partial t} A_{s}(t) c
$$

where $\partial V_{0} / \partial t$ is $\partial V / \partial t$ in the limit $t \rightarrow 0, A_{I}$ is the initial area of $\mathrm{GaN}$ irradiated by ions (i.e., the rectangle around the pillar seen in Fig. 2), $A_{d 1}$ is the projected area of the growing Ga cap in the plane of $A_{I}$, and $A_{d 1} / A_{I}$ is the fraction of $A_{I}$ occupied by the growing cap. The excess Ga generation rate is proportional to $\left(1-A_{d 1} / A_{I}\right)$ because the Ga cap resides on top of the $\mathrm{GaN}$ substrate and masks it from ion irradiation. The last term, $(\partial \gamma / \partial t) A_{s}(t) c$, is the net volumetric $\mathrm{Ga}$ removal rate, expressed as a product of the flux of Ga sputtered from the cap, $\frac{\partial \gamma}{\partial t},\left(\right.$ atoms $\left./ \mathrm{m}^{2} / \mathrm{s}\right), A_{s}$ and the volume of a $\mathrm{Ga}$ atom, $c$.

$\partial V_{0} / \partial t$ was deduced experimentally from the slope of $V(t \rightarrow 0)$, seen in Fig. 3(a), and $\partial \gamma / \partial t$ is a fitting parameter. Solutions to Eq. (1) yield the time evolution of the cap volume $V(t)$ and the corresponding cap diameter $d_{1}(t)$. Figure 3(a) shows the best fit to experiment obtained by setting $\partial \gamma / \partial t$ to $10^{7} \mu \mathrm{m}^{-2} \mathrm{~s}^{-1}$ which corresponds to a realistic sputter yield of $\sim 11$ (Ga atoms per ion incident onto the spherical cap). The calculated $V(t)$ profile is sublinear due to two effects: (i) an increase in $A_{s}$ which acts as a Ga sink, and (ii) a decrease in the size of the Ga source caused by the growth of $A_{d 1}$. This simple time evolution of the size of the $\mathrm{Ga}$ source and sink yields cap growth kinetics that are in agreement with experiment at short growth times. However, the model overestimates the cap growth rate at long times. This divergence is attributed to Ga consumption in sheath formation. Excess Ga is generated by ion irradiation of the GaN substrate and supplied to the cap through diffusion along the surface of the growing sheath. Hence, the fraction of Ga consumed in sheath formation and the $\mathrm{Ga}$ flow rate into the cap increases and decreases with time, respectively. 


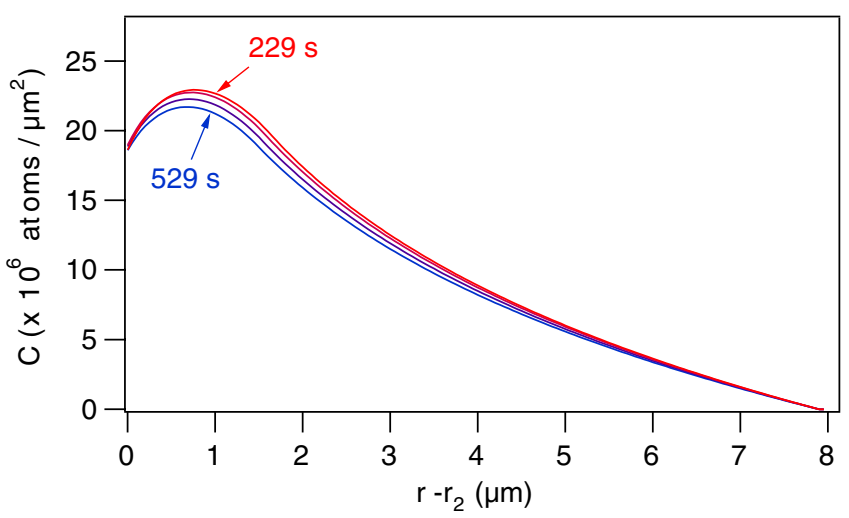

FIG. 6 (color online). Excess Ga concentration $(C)$ along the $\mathrm{GaN}$ substrate versus distance from the pillar base $\left(r-r_{2}\right)$, plotted for $t=229,329,429$, and 529 s. $\left[r_{2}=d_{2} / 2\right]$.

In order to account for sheath formation, we use Eq. (1) to define initial conditions for an explicit simulation of excess Ga generation, diffusion, consumption, and removal from the system. Specifically, we calculate the timedependent excess gallium concentration $C$ across the sheath and the GaN substrate by solving

$$
\frac{\partial C}{\partial t}=a \hat{f}-b C+D \nabla^{2} C
$$

where $a$ is the excess gallium generation coefficient (atoms per ion incident onto GaN), $\hat{f}$ is the mean ion beam flux in the plane of the substrate, $b$ is the Ga uptake rate by the sheath, and $D$ is the Ga diffusion coefficient. A detailed description of the model implementation is provided in Ref. [13].

Equation (2) was solved numerically, using input parameters that are equivalent to those used in Eq. (1), and by using $b$ as a fitting parameter. Figure 3(a) shows plots of $d_{1}, d_{2}$, and $V$ calculated as a function of time. Part (b) of the figure shows corresponding plots of the fraction of excess Ga that is consumed by the sheath and the cap, and the fraction lost through out diffusion (i.e., diffusion away from the region of $\mathrm{GaN}$ bombarded by Ga ions). The latter is approximately constant during growth, thereby validating assumption (ii) made in setting up Eq. (1).

The modeling data in Fig. 3(a) are in excellent agreement with experiment, and were obtained by setting $b$ to $1.1 \times 10^{6} \mathrm{~s}^{-1}$, which yields a physically realistic sheath with a density that is approximately half that of single crystal $\mathrm{GaF}_{3}$ [13]. Solutions to Eq. (2) consistently predict a peak in $C$ on the $\mathrm{GaN}$ substrate, near the pillar base. This peak, shown in Fig. 6, is in the vicinity of "droplet 3" marked on Fig. 2. Such droplets are often observed to nucleate immediately after pillar growth has terminated, and cause nucleation of neighboring pillars a number of which are seen in Fig. 1(e). The frequent nucleation of such droplets is likely caused by a combination of the elevated
Ga concentration, and the presence of an asperity at the pillar base.

We can now combine the above experimental and simulation data to form a detailed picture of pillar growth. Liquid droplets form at the substrate, as in prior studies of III-V semiconductors performed in vacuum $[2,8,9,12]$. $\mathrm{XeF}_{2}$ gives rise to the formation of solid sheaths which deform the growing $\mathrm{Ga}$ droplets into the pillar cores seen in Fig. 4. Pillars emerge through a self-ordering cycle of droplet growth and sheath formation, in which vertical growth occurs only when the cap diameter increases with time. Sheath formation is induced by ion bombardment of the GaN substrate. The absence of $\mathrm{N}$ (within the EDS detection limit) shows that the sheaths are not comprised primarily of material that is sputtered from $\mathrm{GaN}$ and redeposited on the growing pillars. Instead, the most likely mechanism is (i) adsorption of $\mathrm{XeF}_{2}$ gas molecules to the pillar sidewall, (ii) dissociation of the adsorbates by electrons [7] emitted from $\mathrm{GaN}$ due to ion impact, and (iii) formation of gallium fluoride through reactions between liquid gallium and fluorine adsorbates. The abruptness of the core-sheath interface seen in Fig. 4(d) indicates that the sheath constituents are not soluble in Ga. Gallium is supplied through surface diffusion, and generated by ion beam decomposition of the GaN surface. The simulations show that the pillar growth rate is given by the rates of Ga supply through surface diffusion, Ga consumption in sheath formation and Ga removal through sputtering. Sputtering also serves to remove all species, including $\mathrm{F}$, $\mathrm{N}$, and $\mathrm{O}$, through ion bombardment of unmasked regions of the pillar and the substrate. Ion beam heating may assist in maintaining the $\mathrm{Ga}$ in a liquid state, and may alter Ga diffusivity. It is, however, not a prerequisite for pillar formation. Ga is a supercooled liquid at room temperature [16], and our simulation results are insensitive to the exact value of $D$ and account for pillar formation in the absence of temperature gradients caused by localized heating.

*Milos.Toth@uts.edu.au

[1] G. M. Whitesides and B. Grzybowski, Science 295, 2418 (2002); R. M. Bradley and P. D. Shipman, Phys. Rev. Lett. 105, 145501 (2010); S. Facsko, T. Dekorsy, C. Koerdt, C. Trappe, H. Kurz, A. Vogt, and H. L. Hartnagel, Science 285, 1551 (1999).

[2] Q. Wei, J. Lian, W. Lu, and L. Wang, Phys. Rev. Lett. 100, 076103 (2008).

[3] J. V. Barth, G. Costantini, and K. Kern, Nature (London) 437, 671 (2005); W. Lu and C. M. Lieber, Nat. Mater. 6, 841 (2007).

[4] K. W. Kolasinski, Curr. Opin. Solid State Mater. Sci. 10, 182 (2006).

[5] S. Kucheyev, J. Williams, and S. Pearton, Mater. Sci. Eng. R 33, 51 (2001). 
[6] A. Datta, S. Dhara, S. Muto, C. Hsu, C. Wu, C. Shen, T. Tanabe, T. Maruyama, K. Chen, L. Chen, and Y. Wang, Nanotechnology 16, 2764 (2005).

[7] I. Utke, P. Hoffmann, and J. Melngailis, J. Vac. Sci. Technol. B 26, 1197 (2008).

[8] J. H. Wu, W. Ye, B. L. Cardozo, D. Saltzman, K. Sun, H. Sun, J. F. Mansfield, and R. S. Goldman, Appl. Phys. Lett. 95, 153107 (2009).

[9] K. A. Grossklaus and J. M. Millunchick, J. Appl. Phys. 109, 014319 (2011).

[10] L. A. Christel and J. F. Gibbons, J. Appl. Phys. 52, 5050 (1981); S. O. Kucheyev, J.S. Williams, C. Jagadish, J. Zou, V.S. J. Craig, and G. Li, Appl. Phys. Lett. 77, 1455 (2000); S. Kucheyev, J. Williams, J. Zou, C. Jagadish, and G. Li, Appl. Phys. Lett. 77, 3577 (2000).
[11] S. S. Elovikov, I. K. Khrustachev, A.S. Mosunov, and V. E. Yurasova, Radiat. Eff. Defects Solids 158, 573 (2003).

[12] B. K. Chakraverty, J. Phys. Chem. Solids 28, 2413 (1967).

[13] See Supplemental Material at http://link.aps.org/ supplemental/10.1103/PhysRevLett.111.135503 for electron image movies of the growth process and detailed descriptions of the experimental and modelling methodologies.

[14] S.-E. Wu, C.-P. Liu, T.-H. Hsueh, H.-C. Chung, C.-C. Wang, and C.-Y. Wang, Nanotechnology 18, 445301 (2007).

[15] A. S. Barriere, G. Couturier, G. Gevers, H. Guegan, T. Seguelond, A. Thabti, and D. Bertault, Thin Solid Films 173, 243 (1989).

[16] Y. Wang and S. J. Lin, Phys. Rev. B 53, 6152 (1996). 